\title{
Vejez y cáncer de mama, el desafío del siglo 21
}

\author{
CÉSAR SÁNCHEZ R.
}

Due to the increase in life expectancy in Chile, more than $10 \%$ of the population is 60 years or older. Since the incidence of most cancers increases with age, one of every two men and one in three women will develop a malignancy during their lifetime. In Chile breast cancer is the first leading cause of death from cancer among women. Its detection in postmenopausal women has steadily increased since the eighties, due to the expanded use of mammography. Less than 10\% of patients participating in randomized controlled trials for the treatment of breast cancer, are older than 60 years, despite the fact that biological characteristics of breast cancer in older women are different and that most patients with breast cancer are of that age. Due to the high incidence of estrogen receptor positive tumors in this age group, most patients are candidates for hormone therapy. However, in those who need chemotherapy, therapeutic decisions are based on the existence of concomitant diseases and the tolerance to cytotoxic therapy. We review the relationship between age and cancer, the particular characteristics of breast cancer in older women and the alternatives of treatment with chemotherapy in advanced disease.

(Rev Med Chile 2012; 140: 649-658).

Key words: Aged; Antineoplastic agents; Breast, neoplasms.

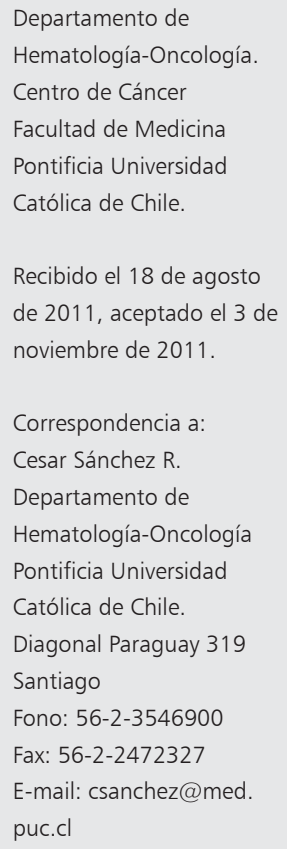

$\mathrm{N}$ unca antes en el mundo hubo tantos adultos mayores (AM). En nuestro país la esperanza de vida al nacer es de casi 75 años para los hombres y de 79 años para las mujeres. Los AM son el grupo etario que proporcionalmente más ha crecido: anualmente 3,3\% comparado a 2,1\% de crecimiento en la población general; el año 2025 en Chile existirán 110 personas mayores de 60 años por cada 100 personas menores de 15 años ${ }^{1}$.

La edad es el principal factor de riesgo para el desarrollo de cáncer de mama (CM), afectando una de cada 8 mujeres a lo largo de su vida. $\mathrm{Al}$ diagnóstico la mitad de las pacientes son mayores de 65 años y 35\% mayores de 70 años ${ }^{2}$. En Chile el CM ocupa la primera causa de muerte asociada a cáncer en mujeres ${ }^{3}$, siendo la mortalidad por $\mathrm{CM}$ entre los 55 y los 84 años superior a la mortalidad atribuible a enfermedades cardiovasculares ${ }^{4}$.

Menos de 10\% de pacientes ingresados a ensa- yos clínicos son $\mathrm{AM}^{5}$ e indicaciones de tratamiento en AM son derivadas de la experiencia en pacientes jóvenes posmenopáusicas. Cambios biológicos y clínicos asociados al envejecimiento afectan el comportamiento y manejo del cáncer ${ }^{6,7}$.

Analizaremos:

- Relación entre edad y cáncer.

- Características propias del CM en el AM.

- Alternativas e indicaciones de tratamiento en AM con CM metastásico (CMM).

\section{Edad y cáncer}

La incidencia de neoplasias aumenta exponencialmente en las últimas décadas de la vida, con un riesgo acumulativo de 1 en 2 para hombres y 1 en 3 para mujeres ${ }^{2}$. Esto se debe principalmente a la aparición de neoplasias epiteliales, contrario a lo que sucede en jóvenes donde predominan cánceres 
mesenquimáticos y hematopoyéticos ${ }^{8}$. Varios factores explican el aumento de las neoplasias con la edad $^{8-12}$ (Tabla 1). Síndromes genéticos asociados a envejecimiento acelerado (progeria) se asocian a mayor riesgo de cáncer, por tanto, genes que determinan procesos de envejecimiento/longevidad, están relacionados a la aparición de neoplasias?.

El daño acumulativo del ADN por radicales libres y radiaciones ionizantes, aumentaría la posibilidad de mutaciones somáticas que superan el umbral carcinogénico ${ }^{8}$. Sin embargo, in vitro, el índice de mutaciones espontáneas es de 2 por 10 elevado a $-7^{13}$, una velocidad insuficiente para desarrollar cáncer en modelos biológicos. La edad también alteraría la capacidad reparativa del $\mathrm{ADN}^{14,15}$.

El telómero humano está compuesto de una secuencia de 6 nucleótidos repetidos cientos a miles de veces en el extremo final de los cromosomas y sintetizados por una transcriptasa reversa llamada telomerasa. Durante cada división celular los telómeros se acortan. La aparición de la telomerasa durante el proceso carcinogénico mantiene la longitud del telómero incrementando la capacidad replicativa de la célula ${ }^{16}$. El fibroblasto humano normal puede dividirse 50 a 60 veces (límite de Hayflick), luego de esto su viabilidad comienza a disminuir. Las células somáticas luego de un número finito de divisiones celulares entran a un
Tabla 1. Cambios epiteliales asociados al envejecimiento que predisponen a la aparición de cáncer

\begin{tabular}{|l|}
\hline Acumulación de mutaciones \\
\hline Alteración de los mecanismos de reparación \\
\hline Inestabilidad genómica \\
\hline Crisis del telómero \\
\hline Metilación del ADN \\
\hline Senescencia celular \\
Inflamación crónica \\
\hline
\end{tabular}

proceso irreversible de detención del crecimiento llamado senescencia replicativa. Si mutaciones somáticas impiden que la célula senescente salga del ciclo celular, las divisiones celulares mantenidas, asociadas a una disfunción del telómero, ocasionarían gran inestabilidad genómica conocida como "crisis del telómero" 17 . Por otro lado, este fenómeno de senescencia celular aumenta la resistencia a la apoptosis ${ }^{16}$ (Figura 1).

La senescencia celular puede contribuir al crecimiento reducido de tumores, lenta diseminación de neoplasias ${ }^{6,12}$ y menor respuesta a factores angiogénicos, esto explicaría el mejor pronóstico de algunos tumores en AM; por

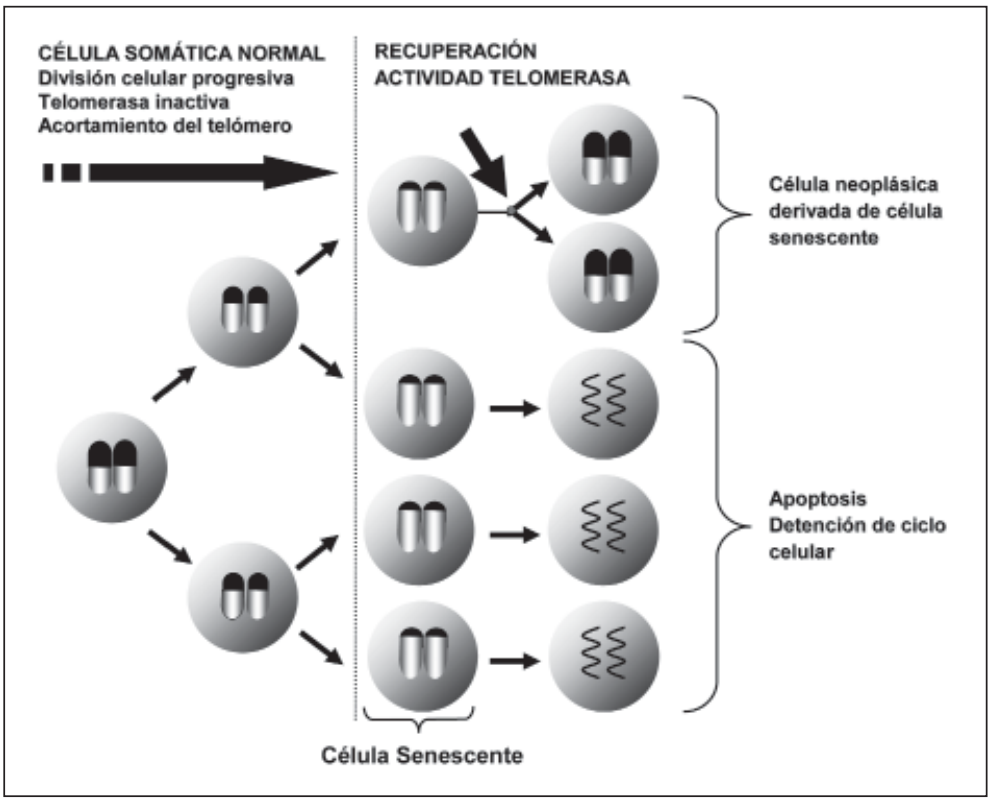

Figura 1. Acortamiento del telómero y cáncer. Células normales, sin actividad de telomerasa, sufren progresivo acortamiento del telómero en cada división celular. La actividad de telomerasa es capaz de mantener la longitud del telómero. 
otro lado, la declinación en la respuesta inmune observada con el envejecimiento favorecería el desarrollo de neoplasias debido a la tolerancia inmune $\mathrm{e}^{18-20}$.

Mecanismos epigenéticos, como la hipermetilación de islas CpG (citosina-guanina) cercana a zonas promotoras, han mostrado ser un mecanismo común de silenciamiento de genes supresores de tumores progresivo con la edad ${ }^{21}$.

\section{Características del CM en el AM}

En algunos cánceres, la edad determina la presentación clínica y la respuesta al tratamiento. En pacientes AM con leucemia mieloide aguda las posibilidades de remisiones mantenidas son inferiores dado el componente de mielodisplasia basal, expresión de genes de resistencia a drogas y acumulación de cambios genéticos en los blastos leucémicos ${ }^{22}$.

Para el CM algunas revisiones muestran que menor cantidad de pacientes AM se diagnostican en etapas iniciales. La histología más frecuente en AM, al igual que en jóvenes, es el cáncer ductal infiltrante, pero aparecen con mayor frecuencia histologías de tipo mucinoso y papilar. Mientras los carcinomas mucinosos, explican sólo el 1\% de los cánceres en mujeres premenopáusicas, corresponden al 4-5\% en mujeres de 75-85 años. Del mismo modo 0,3\% de los cánceres en mujeres premenopáusicas tienen una histología papilar, mientras casi $1 \%$ de AM se presentan de este modo $^{6,23}$. La expresión del RE en el tumor es $46 \%$ en mujeres menores de 35 años y $82 \%$ en mayores de 65 años $^{24}$. El Her2/neu, miembro de una familia de genes que codifican receptores transmembrana para factores de crecimiento, está amplificado y sobreexpresado con mayor frecuencia en tumores mal diferenciados, con compromiso nodal y confiere resistencia relativa a QT y terapia hormonal $(\mathrm{TH})^{25,26}$. Mientras 20-25\% de pacientes jóvenes tiene sobreexpresión del receptor, menos de 15\% de AM presenta esta característica ${ }^{25}$.

También la tasa de proliferación celular del tejido tumoral (estudio de fase $\mathrm{S}$ : fase de síntesis) disminuye con la edad ${ }^{6}$.

Todo esto refleja que el CM en AM tiene con mayor frecuencia un perfil endocrino respondedor (Tabla 2); por lo tanto, la mayoría reciben $\mathrm{TH}$ durante su evolución.

\section{Tratamiento del CMM en el AM}

La sobrevida global (SG) de pacientes con CM es de $90 \%$ a 5 años. En pacientes con CMM la sobrevida a 5 años es $20 \%$, con una mediana de 2 a 4 años ${ }^{2}$. Sin embargo, pacientes jóvenes, con intervalo libre de enfermedad mayor a un año, sin compromiso visceral y que logran remisión completa, pueden alcanzar una SG de $10 \%$ a 10 años. Al contrario, aquellas pacientes con metástasis hepáticas o de sistema nervioso central no superan una mediana de sobrevida de seis meses ${ }^{27}$. Las indicaciones de tratamiento del CMM en el AM se extrapolan de algoritmos definidos para mujeres posmenopáusicas ${ }^{6}$. Hutchins y cols analizaron da-

\section{Tabla 2. Clasificación de pacientes con cáncer de mama metastásico según expresión de receptores por inmunohistoquímica}

RE y/o RP positivos (endocrino respondedores)

Sobre-expresión de Her 2 Neu (utilidad de anticuerpos monoclonales contra HER 2: Trastuzumab)

Triple negativos: RE, RP y Her 2 Neu negativos

Tabla 3. Mecanismos de acción del receptor de estrógenos

\begin{tabular}{ll}
$\begin{array}{l}\text { Mecanismos } \\
\text { de acción }\end{array}$ & Unión a elementos respondedores a \\
Genómicos & $\begin{array}{l}\text { estrógenos en regiones del promotor } \\
\text { Interacción con otros factores de trans- } \\
\text { cripción }\end{array}$ \\
\hline No genómicos & Interacción y activación de kinasas.
\end{tabular}

Tabla 4. Potenciales mecanismos de resistencia a TH

Pérdida de expresión o mutación del RE

Falta de expresión del RP

Aumento de expresión del RE beta

Metabolismo de agentes hormonales

Aumento de co-reguladores

Aumento de la sensibilidad a estrógenos

Aumento de señales de factores de crecimiento 


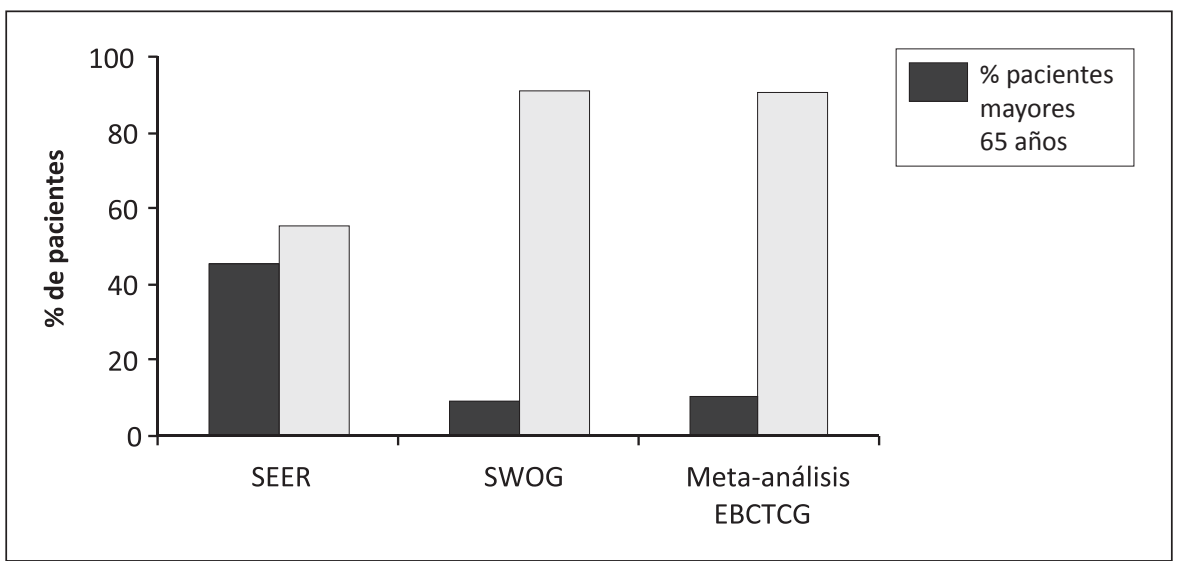

Figura 2. Sub-representación de pacientes con cáncer de mama en estudios del SWOG y metaanálisis del EBCTCG. tos de 16.396 pacientes oncológicos enrolados en protocolos clínicos del SWOG (Southwest Oncology Group). Compararon la edad de estos pacientes con la de pacientes con cáncer en Estados Unidos de Norteamérica (registros del SEER). La subrepresentación de pacientes mayores de 65 años fue marcada en pacientes con CM: de los pacientes enrolados en estudios clínicos, $9 \%$ tenía más de 65 años comparado con $45 \%$ en los registros del SEER $(\mathrm{p}<0,001)^{5}$. Las recomendaciones de QT adyuvante derivadas del metaanálisis del EBCTCG no incluyen a este grupo etario y la cantidad de pacientes mayores de 70 años analizada es inferior al $10 \%{ }^{28}$ (Figura 2).

\section{Terapia hormonal}

La QT muestra mayores tasas de respuesta que la TH y en un plazo más abreviado; sin embargo, el uso inicial de $\mathrm{TH}$ no altera la $\mathrm{SG}^{29}$. En mujeres posmenopáusicas la supresión estrogénica se logra a través de: modulación de la actividad del RE (tamoxifeno, raloxifeno), degradación del RE (fulvestrant); o reducción de la producción de estrógenos en tejidos periféricos por medio de inhibidores de aromatasa (IA). Para pacientes con RE y/o RP (receptores de progesterona) positivos la terapia inicial es TH, excepto si hay rápida progresión o metástasis viscerales (hígado, pulmón) ${ }^{23,27}$ (Tabla 5).

Aun en pacientes con CMM no endocrino respondedor y sin compromiso visceral, se puede considerar un "curso de prueba" de terapia endocrina ${ }^{30}$.

El tamoxifeno ha sido la TH clásica del CM hace 30 años, logrando respuestas en hasta $80 \%$ de los pacientes con RE/RP+; sin embargo, el 50\% de las pacientes con enfermedad avanzada no responden al tratamiento y casi $40 \%$ de las pacientes que reciben tamoxifeno como tratamiento adyuvante tienen recaídas o mueren por la enfermedad. Recientemente los IA, han mostrado niveles de efectividad superior o similar al tamoxifeno ${ }^{31,32}$.

Los efectos adversos del tamoxifeno son aumento de riesgo de cáncer de endometrio, tromboembolismo venoso y cataratas, entre otros ${ }^{33}$.

Estos riesgos son mayores en AM. Sin embargo, el riesgo de mortalidad combinada secundaria a cáncer de endometrio y tromboembolismo es menor al $1 \%$.

Los efectos adversos principales de los IA son el aumento de la osteoporosis y mialgias. Se ha propuesto el uso de bifosfonatos para evitar el efecto óseo adverso de la supresión estrogénica producida por IA; sin embargo, no hay reportes de la toxicidad y los efectos del uso prolongado de bifosfonatos en AM: disminución de la filtración glomerular y osteonecrosis de mandíbula ${ }^{34,35}$.

Tabla 5. Clasificación de CMM según sitio de enfermedad metastásica

\begin{tabular}{|c|c|}
\hline $\begin{array}{l}\text { Sitio de enfermedad } \\
\text { metastásica }\end{array}$ & \\
\hline Sin crisis visceral & $\begin{array}{l}\text { Metástasis tejidos blandos } \\
\text { (piel, celular subcutáneo), } \\
\text { ganglionar, compromiso óseo }\end{array}$ \\
\hline Con crisis visceral & $\begin{array}{l}\text { Metástasis parénquimas: } \\
\text { pulmonar, hepático, sistema } \\
\text { nervioso central }\end{array}$ \\
\hline
\end{tabular}


Vejez y cáncer de mama - C. Sánchez

Tabla 6. Tipos de hormonoterapia en pacientes con CM

\begin{tabular}{|llll|}
\hline $\begin{array}{l}\text { Moduladores selectivos del } \\
\text { receptor de estrógenos }\end{array}$ & $\begin{array}{l}\text { Inhibidores de } \\
\text { Aromatasa }\end{array}$ & Anti-estrógenos & Otros \\
\hline Tamoxifeno & Anastrozol & Fulvestrant & Andrógenos \\
\hline Raloxifeno & Letrozol & & Estrógenos \\
& Exemestane & Acetato de megestrol \\
\hline
\end{tabular}

Para pacientes que progresan bajo $\mathrm{TH}$ de primera línea, existen varias alternativas endocrinas, efectivas especialmente para aquellos con enfermedad metastásica ósea o en tejido blando, respuesta larga a $\mathrm{TH}$ previa y/o que recaen luego de un año ${ }^{23,36}$ (Tabla 6).

\section{Cáncer, quimioterapia y edad}

A pesar del éxito de la TH algunas pacientes necesitan QT (Tabla 7). A diferencia de las terapias endocrinas con un blanco molecular específico, la QT convencional citotóxica no tiene un efecto selectivo.

El envejecimiento está asociado a una declinación progresiva de la reserva funcional de múltiples órganos, lo que afecta las propiedades farmacocinéticas y farmacodinámicas de las drogas y disminuye la tolerancia al daño del tejido normal $^{37}$ (Tabla 8). El cambio farmacocinético más importante con la edad es el cambio del volumen de distribución $(\mathrm{Vd})$ y la excreción renal. $\mathrm{La}$ disminución del agua corporal reduce el Vd para drogas hidrosolubles, la caída en la albúmina y en la hemoglobina puede también restringir el Vd y aumentar la toxicidad de la QT. De estos factores la anemia es el único que podemos corregir ${ }^{38}$.

La edad disminuye la masa y el flujo sanguíneo hepático, además la función hepática puede alterarse por la declinación en la actividad de la citocromo P450, el uso de otros medicamentos concomitantes (polifarmacia) puede interactuar con el metabolismo hepático de drogas citotóxicas. Por otro lado, cambios en la absorción intestinal pueden influir en la efectividad de las drogas orales. También cambios farmacodinámicos pueden afectar la toxicidad y actividad de drogas antineoplásicas. La eliminación de monocitos afectados por aductos tras la administración de cisplatino es más lenta en pacientes mayores; este retraso en la reparación del daño al ADN puede aumentar la citotoxicidad derivada de la QT. En algunos pacientes el nivel de enzimas que catabolizan drogas puede estar disminuido, es el caso, por ejemplo, del déficit de dihidropirimidina deshidrogenasa que cataboliza el 5 fluorouracilo, aumentando la probabilidad de mucositis ${ }^{39}$. También cambios farmacodinámicos pueden aumentar la resistencia a las terapias citotóxicas; por ejemplo, la mayor expresión en blastos mieloides de AM de glicoproteína P aumenta la eliminación

Tabla 7. Pacientes con CMM que necesitan quimioterapia

\begin{tabular}{|l|}
\hline Tumor sin expresión de RE/RP \\
\hline No expresión de HER-2 \\
Resistencia al tratamiento endocrino \\
Crisis visceral \\
Enfermedad metastásica extensa \\
\hline Intervalo libre de enfermedad menor a 1 año \\
\hline
\end{tabular}

Tabla 8. Cambio farmacocinéticas y farmacodinámicos asociados al envejecimiento que afectan uso de quimioterapia

Disminución de la motilidad intestinal

Disminución del flujo sanguíneo esplácnico

Disminución secreción de enzimas y atrofia de mucosa gastrointestinal

Disminución agua intracelular y aumento de grasa corporal Disminución albúmina sérica (drogas unidas a albúmina)

Anemia

Sistema citocromo P450 y polifarmacia

Caída del clearence de creatinina

Disminución de células madre (Disminución de Stem cells) 
desde el interior de la célula de drogas de origen natural. Las células tumorales de pacientes mayores podrían tener más resistencia a la apoptosis debido a que estas neoplasias derivarían de células senescentes ${ }^{20}$. La hipoxia tumoral por disminución de la angiogénesis puede aumentar la resistencia a agentes alquilantes y radioterapia ${ }^{19}$.

Una disminución de las stem cells con el envejecimiento contribuye a la disminución de la reparación de tejidos normales como el tejido hematopoyético y las mucosas, y a la mayor susceptibilidad del tejido normal a la toxicidad de agentes antineoplásicos ${ }^{19}$; por lo que cualquier noxa y pérdida adicional puede comprometer la función (cardiomiopatía, neurotoxicidad) ${ }^{37-40}$.

La incidencia de anemia, neutropenia y plaquetopenia secundarias a la QT son más comunes, más severas, asociadas a mayor número de infecciones, hospitalizaciones y mayor mortalidad en el AM. La menor tolerancia a la QT convencional en el AM también ha inducido a disminuir las dosis en terapias adyuvantes demostradamente útiles en algunas patologías oncológicas curables, lo que podría explicar en algunos casos el peor pronóstico de AM sub-tratados ${ }^{39}$.

A pesar de esto, estudios Fase III en AM seleccionados, en buenas condiciones generales, en instituciones especializadas y con dosis ajustadas, reportan toxicidad de la QT no diferente al paciente joven ${ }^{41,42}$.

\section{Evaluación geriátrica y quimioterapia. Co-morbilidades y estado funcional}

El envejecimiento es un proceso individual en términos de expectativa de vida, reserva funcional, soporte social y preferencias personales ${ }^{43}$. Cualquier tratamiento en CMM es paliativo y su principal objetivo es mantener o mejorar la calidad de vida ${ }^{27}$. El principal desafío en el manejo de AM con cáncer es evaluar si los beneficios del tratamiento son superiores al riesgo de complicaciones.

Al decidir cualquier tratamiento debemos intentar responder:

¿El paciente morirá de cáncer o con cáncer?, ¿Sufrirá complicaciones derivadas del cáncer?, ¿Proveerá el tratamiento más beneficio que daño?

La edad cronológica y los exámenes de laboratorio son limitados en esta evaluación. La prevalencia de cambios relacionados a la edad aumenta después de los 70 años ${ }^{43}$. Noventa porciento de las personas con signos de envejecimiento tienen sobre 70 años. Luego de los 85 años las personas frágiles aumentan, tienen un rápido deterioro de su capacidad visual y auditiva, son más susceptibles a accidentes y presentan mayor tendencia a la dependencia funcional.

Un instrumento propuesto para estimar la reserva funcional y la expectativa de vida es una evaluación multidimensional del envejecimiento conocida como CGA (Comprehensive Geriatric Assessment $)^{43,44}$. El CGA incluye: evaluación de comorbilidades, condiciones socioeconómicas como transporte, cuidados domiciliarios y facilidad de ayuda en caso de urgencias; evaluación de dependencia funcional, de condiciones emocionales y cognitivas, y estimación de expectativa de vida.

Sin embargo, no existen estudios de distribución aleatoria que demuestren la utilidad de esta escala de evaluación o similares, a pesar de que el sentido común y estudios observacionales lo apoyan $^{44-46}$ (Tabla 9).

Los instrumentos clásicos de medición de capacidad funcional: Karnofsky, ECOG no son buenos predictores en el anciano ${ }^{43}$. Ciertas co-morbilidades conllevan un alto riesgo de mortalidad. Por ejemplo, una mujer con CM no metastásico y tres o más co-morbilidades serias tiene 20 veces más probabilidades de morir en los siguientes tres años por causas distintas al cáncer ${ }^{47}$. Algunas co-morbilidades específicas pueden influir en la decisión del mejor tratamiento o pueden resultar en reducción de las dosis de QT. Por ejemplo, la alteración de la función renal puede limitar el uso de esquemas como CMF (ciclofosfamida, metotrexate, fluorouracilo) y contraindicar el uso de platino ${ }^{39,42}$.

\section{Tabla 9. Recomendaciones al evaluar uso de quimioterapia en pacientes}

\section{Evaluación geriátrica en mayores de 70 años}

Dosis según clearence de creatinina

Factor estimulante de colonias profiláctico en quimioterapias tipo CHOP

Mantener niveles de hemoglobina mayor a $12 \mathrm{~g} / \mathrm{dL}$

Considerar alternativas a las antraciclinas 
Vejez y cáncer de mama - C. Sánchez

\section{Uso de quimioterapia en la paciente mayor}

La edad por sí sola no contraindica el uso de tratamientos que mejoren la calidad de vida o extiendan las posibilidades de vivir. La experiencia de Piedmont, en un estudio comparativo, muestra que AM con buena capacidad funcional, utilizando dosis de QT corregidas según clearence de creatinina, presentan toxicidades que no difieren de la reportada en pacientes jóvenes ${ }^{41}$.

Se han desarrollado estrategias para proteger al paciente, como por ejemplo el uso de cardio y nefroprotectores, ajuste de dosis o cálculo de la dosis con el área bajo la curva tiempo-concentración (carboplatino por ejemplo) ${ }^{48}$ (Tabla 9).

No existe un esquema de QT estándar para el CMM y las guías clínicas de manejo de esta enfermedad sugieren varias alternativas ${ }^{49}$ (Tabla 10 y 11$)$.
En CMM las drogas más activas son las antraciclinas y los taxanos. Como agentes únicos producen respuesta en $20-80 \%$ de las pacientes ${ }^{50-53}$ (Tabla 10).

Fossatti, en una revisión sistemática de 189 estudios aleatorizados realizados entre 1975-1997, mostro que la SG es superior para la poli-quimioterapia versus monoterapia ( $\mathrm{HR}=0,82 ; 95 \%$ IC, $0,75$ a 0,90$)$; sin embargo, la calidad de vida se midió sólo en $9,5 \%$ de las pacientes, los trabajos incluidos en esta revisión son pequeños, no dirigidos a AM y con esquemas de QT actualmente poco utilizados ${ }^{54}$.

En pacientes frágiles el alivio de los síntomas y la mantención de la calidad de vida son fundamentales, algunas terapias citotóxicas con bajos niveles de complicaciones tales como gemcitabina, navelbine o taxanos semanales (Tabla 12) son

\section{Tabla 10. Agentes quimioterápicos y toxicidad en adulto mayor}

\begin{tabular}{|c|c|}
\hline Droga & Toxicidad \\
\hline Antraciclinas & $\begin{array}{l}\text { Toxicidad cardiaca, mielodepresión, alopecia, náuseas, vómitos } \\
\text { Precaución en pacientes cardiópatas, hipertensos, radioterapia mediastino previa. Vigilar dosis acumulativa }{ }^{48}\end{array}$ \\
\hline Taxanos & $\begin{array}{l}\text { Mielodepresión cuando se usa cada tres semanas } \\
\text { Neuropatía (paclitaxel), edema (docetaxel) en uso semanal } \\
\text { Contraindicado en pacientes con alteraciones de la conducción auriculoventricular. Su uso requiere preme- } \\
\text { dicación con corticoides }{ }^{51,52}\end{array}$ \\
\hline Vinorelbine & Mielodepresión, neuropatía 49,50 \\
\hline Gemcitabina & Trombopenia ${ }^{54}$ \\
\hline Capecitabina & $\begin{array}{l}\text { Mucositis, síndrome mano-pie. Control médico semanal al inicio del tratamiento. Corregir dosis según } \\
\text { función renal }{ }^{53}\end{array}$ \\
\hline Trastuzumab & Reacciones alérgicas, cardiotoxicidad. Control periódico de función cardiaca \\
\hline
\end{tabular}

Tabla 11. Recomendaciones generales al iniciar quimioterapia en el adulto mayor

Evaluar estado funcional. El performance status aislado en pacientes mayores no es útil

Analizar presencia de co-morbilidades

Red de apoyo: social, familiar, transporte, dependencia

Sitio y extensión de compromiso metastásico, riesgo vital

Expectativas de vida

Utilizar monoterapia

Cuidados de soporte: Mantener hemoglobina sobre $12 \mathrm{~g} / \mathrm{dl}$

Uso de factor estimulante de colonias en riesgo de neutropenia (quimioterapia combinada, radioterapia previa extensa, compromiso óseo)

Bifosfonatos-Denosumab: Hipercalcemia, metastásis óseas 
Tabla 12. Algoritmo de manejo CMM mujer mayor de 65 años

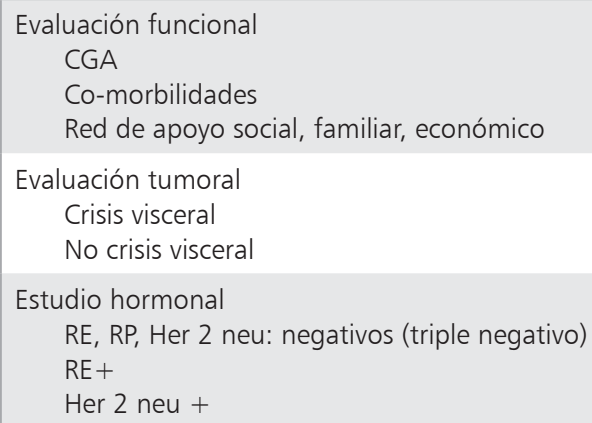

Evaluación tumoral Crisis visceral

No crisis visceral

Estudio hormonal

$R E, R P$, Her 2 neu: negativos (triple negativo) $\mathrm{RE}+$

Her 2 neu +

Monoterapia según co-morbilidades y preferencia del paciente

CGA: Comprehensive geriatric assessment.

buenas alternativas. El trastuzumab, un anticuerpo monoclonal anti HER2, tiene un bajo perfil de toxicidad, respuesta como monoterapia de $20 \%$ y en combinación con QT de 30-70\%. Puede producir cardiotoxicidad, generalmente reversible, por lo que debe vigilarse periódicamente la función $\operatorname{cardiaca}^{55}$ (Tabla 10).

\section{Cuidados de soporte médico}

Metástasis óseas se presentan hasta en $80 \%$ de las pacientes y frecuentemente cursan con complicaciones: dolor, fracturas, compresión medular e hipercalcemia. Los bifosfonatos y agentes modificadores del metabolismo óseo han demostrado disminuir la incidencia de dolor y complicaciones esqueléticas, cuando se agregan a TH o QT ${ }^{56}$. Muchos pacientes AM tienen un deterioro de la función renal por lo que tienen un riesgo mayor de nefrotoxicidad asociada al uso de bifosfonatos endovenosos. Los bifosfonatos orales, al igual que el denosumab de uso subcutáneo, no son asociados con efectos adversos en la función renal, y más aun no necesitan visitas al hospital, lo que es especialmente importante en pacientes con dificultades en transporte y movilización ${ }^{56-59}$. El riesgo de osteonecrosis de mandíbula también podría estar aumentado en pacientes AM, ya que las infecciones y procedimientos dentales; más frecuentes en este grupo; son un factor de riesgo para la aparición de esta complicación. La anemia asociada al cáncer y la QT puede requerir uso de eritropoyetina $^{60}$ (Tabla 9).

\section{Conclusiones}

El cáncer se ha transformado en la principal causa de muerte en hombres y mujeres entre los 60-79 años de edad ${ }^{2}$.

Se estima que el año 2034 existirán la misma cantidad de AM que menores de 15 años $^{61,62}$. No disponemos de instrumentos validados para identificar con certeza aquellos AM de alto riesgo para desarrollar efectos tóxicos derivados de la QT y que por tanto, no se benefician de su prescripción. El objetivo más importante a desarrollar es el diseño de estudios Fase III, que incluyan un número estadísticamente significativo de AM, con objetivos específicos para este grupo etario y participación activa de oncólogos y geriatras experimentados en el cuidado del AM con cáncer.

Las indicaciones de QT en pacientes con CMM deben ser adaptadas al estado funcional y comorbilidades propias de los AM. La utilización de monoterapia es factible y su uso ha sido descrito en estudios fase II y III.

\section{Referencias}

1. Censo de Población y Vivienda. Instituto Nacional de Estadística. 2002.

2. Jemal A, Siegel R, Ward E, Hao Y, Xu J, Murray T, et al. Cancer Statistics 2008. CA Cancer J Clin 2008; 58: 71-96.

3. http://portal.sernam.cl/?m=sp\&i=1723\#. Visitado octubre 2011.

4. Yancik R, Wesley MN, Ries LA, Havlik RJ, Edwards BK, Yates JW. Effect of age and comorbidity in postmenopausal breast cancer patients aged 55 years and older. JAMA 2001; 285: 885-92.

5. Hutchins LF, Unger JM, Crowley JJ, Coltman CA Jr, Albain KS. Underrepresentation of patients 65 years of age or older in cancer- treatment trials. N Engl J Med 1999; 341: 2061-7.

6. Kimmick G, Muss HB. Breast cancer in older patients. Semin Oncol 2004; 31: 234-48.

7. Hanson LC, Muss HB. Cancer in the oldest old: making better treatment decisions J Clin Oncol 2010; 28: 1975-6.

8. Depinho RA. The age of cancer. Nature 2000; 408: 24854.

9. Martin GM, Oshima J. Lessons from human progeroid syndromes. Nature 2000; 408: 263-6.

10. Nowell PC. The clonal evolution of tumor cell populations. Science 1976; 194: 23-8.

11. Vogelstein B, Fearon ER, Hamilton SR, Kern SE, Preisinger AC, Leppert M, et al. Genetic alterations during 
colorectal-tumor development. N Engl J Med 1988; 319: 525-32.

12. Eppenberger-Castori S, Moore DH Jr, Thor AD, Edgerton SM, Kueng W, Eppenberger U, et al. Age-associated biomarker profiles of human breast cancer. Int J Biochem Cell Biol 2002; 34: 1318-30.

13. Oller AR, Rastogi P, Morgenthaler S, Thilly WG. A statistical model to estimate variance in long term-low dose mutation assays: testing of the model in a human lymphoblastoid mutation assay. Mutat Res 1989; 216: 149-61.

14. Ershler WB, Longo DL. Aging and cancer: issues of basic and clinical science. J Natl Cancer Inst 1997; 89: 1489-97.

15. Hanahan D, Weinberg RA. Hallmarks of cancer: the next generation Cell 2011; 144: 646-74.

16. Haber DA. Telomeres, cancer, and immortality. N Engl J Med 1995; 332: 955-6.

17. Harley CB, Futcher AB, Greider CW. Telomeres shorten during ageing of human fibroblasts. Nature 1990; 345: 458-60.

18. Schneider EL, Mitsui Y. The relationship between in vitro cellular aging and in vivo human age. Proc Natl Acad Sci U S A 1976; 73: 3584-8.

19. Holmes FF, Wilson J, Blesch KS, Kaesberg PR, Miller R, Sprott R. Biology of cancer and aging. Cancer 1991; 68: 2525-6.

20. Campisi J. Suppressing cancer: the importance of being senescent. Science 2005; 309: 886-7.

21. Herman JG, BayliN SB. Gene silencing in cancer in association with promoter hypermethylation. $\mathrm{N}$ Engl J Med 2003; 349: 2042-54

22. Stone RM, O'donnell Mr, Sekeres MA. Acute myeloid leukemia. Hematology Am Soc Hematol Educ Program 2004; 98-117.

23. Holmes CE, Muss HB. Diagnosis and treatment of breast cancer in the elderly. CA Cancer J Clin 2003; 53: 227-44.

24. Harvey JM, Clark GM, Osborne CK, Allred DC. Estrogen receptor status by immunohistochemistry is superior to the ligand-binding assay for predicting response to adjuvant endocrine therapy in breast cancer. J Clin Oncol 1999; 17: 1474-81.

25. Burstein HJ. The distinctive nature of HER2-positive breast cancers. N Engl J Med 2005; 353: 1652-4.

26. Slamon DJ, Leyland-Jones B, Shak E, Fuchs H, Paton V, Bajamonde A, et al. Use of Chemotherapy plus a Monoclonal Antibody against HER2 for Metastatic Breast Cancer That Overexpresses HER2. N Engl J Med 2001; 344: 783-92.

27. Bernard-Marty C, Cardoso F, Piccart MJ. Facts and controversies in systemic treatment of metastatic breast cancer. Oncologist 2004; 9: 617-32.
28. Early Breast Cancer Trialists' Collaborative Group (EBCTCG). Effects of chemotherapy and hormonal therapy for early breast cancer on recurrence and 15-year survival: An overview of the randomised trials. Lancet 2005; 365: 1687-717.

29. Taylor SG 4th, Gelman RS, Falkson G, Cummings FJ. Combination chemotherapy compared to tamoxifen as initial therapy for stage IV breast cancer in elderly women. Ann Intern Med 1986; 104: 455-61.

30. Vogel CL, East DR, Voigt W, Thomsen S. Response to tamoxifen in estrogen receptor-poor metastatic breast cancer. Cancer 1987; 60: 1184-9.

31. Pritchard KI. Endocrine therapy of advanced disease: analysis and implications of the existing data. Clin Cancer Res 2003; 9: 460s-7s.

32. Rodríguez Lajusticia L, Martín Jiménez M, LópezTarruella Cobo S. Endocrine therapy of metastatic breast cancer. Clin Transl Oncol 2008; 10: 462-7.

33. Fisher B, Costantino JP, Wickerham DL, Redmond CK, Kavanah M, Cronin WM, et al. Tamoxifen for prevention of breast cancer: report of the National Surgical Adjuvant Breast and Bowel Project P-1 Study. J Natl Cancer Inst 1998; 90: 1371-88.

34. Smith IE, Dowsett M. Aromatase inhibitors in breast cancer. N Engl J Med 2003; 348: 2431-42.

35. Guarneri V, Donati S, Nicolini M, Giovannelli S, D'amico R, Conte PF. Renal safety and efficacy of i.v. bisphosphonates in patients with skeletal metastases treated for up to 10 years. Oncologist 2005; 10: 842-8.

36. Ellis M, Gao F, Dehdashti F, Jeffe D, Marcom P, Carey $\mathrm{L}$, et al. Lower-dose vs high-dose oral estradiol therapy of hormonereceptor-positive, aromatase inhibitorresistant advanced breastcancer: a phase 2 randomized study. JAMA 2009; 302: 774-80.

37. Balducci L, Extermann M, Carreca I. Management of breast cancer in the older woman. Cancer Control 2001; 8: 431-41.

38. Wasil T, Lichtman SM. Clinical pharmacology issues relevant to the dosing and toxicity of chemotherapy drugs in the elderly. Oncologist 2005; 10: 602-12.

39. Balducci L, Beghe C. Pharmacology of Chemotherapy in the Older Cancer Patient Cancer Control 1999; 6: 46670.

40. Doyle JJ, Neugut AI, Jacobson JS, Grann VR, Hershman DL. Chemotherapy and cardiotoxicity in older breast cancer patients: a population-based study. J Clin Oncol 2005; 23: 8597-605.

41. Christman K, Muss HB, Case Ld, Stanley V. Chemotherapy of metastatic breast cancer in the elderly. The Piedmont Oncology Association experience. JAMA 1992; 268: 96-7. 
42. Gelman RS, Taylor SG 4th. Cyclophosphamide, methotrexate, and 5-fluorouracil chemotherapy in women more than 65 years old with advanced breast cancer: the elimination of age trends in toxicity by using doses based on creatinine clearance. J Clin Oncol 1984; 2: 1404-13.

43. Balducci L, Extermann M. Management of Cancer in the Older Person: A Practical Approach. Oncologist 2000; 5: 224-37.

44. Horgan A, Leighl N, Coate L, Liu G, Palepu P, Knox J, Perera N, et al. Impact and Feasibility of a Comprehensive Geriatric Assessment in the Oncology Setting: A Pilot Study. Am J Clin Oncol 2011 Mar 17. [Epub ahead of print].

45. Balducci L, Extermann M. Cancer and aging. An evolving panorama. Hematol Oncol Clin North Am 2000; 14: $1-16$

46. Hurria A, Togawa K, Mohile SG, Owusu C, Klepin HD, Gross CP, et al. Predicting chemotherapy toxicity in older adults with cancer: a prospective multicenter study. J Clin Oncol 2011; 29: 3457-65.

47. Satariano WA, Ragland DR. The effect of comorbidity on 3-year survival of women with primary breast cancer. Ann Intern Med 1994; 120: 104-10.

48. Hensley M, Hagerty K, Kewalramani T, Green D, Meropol N, Wasserman T, et al. American Society of Clinical Oncology 2008 clinical practice guideline update: use of chemotherapy and radiation therapy protectants. J Clin Oncol 2009; 27: 127-45.

49. www.nccn.org/professionals/physician_gls/PDF/breast. pdf. Visitado julio de 2011.

50. Vogel C, O'rourke M, Winer E, Hochster H, Chang A, Adamkiewicz B, et al. Vinorelbine as first-line chemotherapy for advanced breast cancer in women 60 years of age or older. Ann Oncol 1999; 10: 397-402.

51. Sorio R, Robieux I, Galligioni E, Freschi A, Colussi Am, Crivellari D, et al. Pharmacokinetics and tolerance of vinorelbine in elderly patients with metastatic breast cancer. Eur J Cancer 1997; 33: 301-3.

52. Wildiers H, Paridaens R. Taxanes in elderly breast cancer patients. Cancer Treat Rev 2004; 30: 333-42.

53. Bajetta E, Procopio G, Celio L, Gattinoni L, Della Torre $S$, Mariani L, et al. Safety and efficacy of two different doses of capecitabine in the treatment of advanced breast cancer in older women. J Clin Oncol 2005; 23: 2155-61.

54. Fossati R, Confalonieri C, Torri V, Ghislandi E, Penna A, Pistotti V, et al. Cytotoxic and hormonal treatment for metastatic breast cancer: a systematic review of published randomized trials involving 31,510 women. J Clin Oncol 1998; 16:3439-60.

55. Martín M, Esteva FJ, Alba E, Khandheria B, Pérez-Isla L, García-Sáenz JA, et al. Minimizing Cardiotoxicity While Optimizing Treatment Efficacy with Trastuzumab: Review and Expert Recommendations. Oncologist 2009; 14: 1-11.

56. Coleman RE. Bisphosphonates in breast cancer. Ann Oncol 2005; 16:687-95.

57. Henrich D, Bergner R, Hoffmann M, Schanz J, Landmann T, Uppenkamp $M$, et al. Ibandronate in the treatment of hypercalcemia or nephrocalcinosis in patients with multiple myeloma and acute renal failure. Support Care Cancer 2005; 13: 463; 21a-150a

58. Gridelli C. The Use of Bisphosphonates in Elderly Cancer Patients. Oncologist 2007; 12: 62-71.

59. Stopeck A, Lipton A, Body J, Steger G, Tonkin K, De Boer $\mathrm{R}$, et al. Denosumab compared with zoledronic acid for the treatment of bone metastases in patients with advanced breast cancer: a randomized, double-blind study. J Clin Oncol 2010; 28: 5132-9.

60. Leonard RC, Untch M, Von Koch F. Management of anaemia in patients with breast cancer: role of epoetin. Ann Oncol 2005; 16:817-24.

61. Marín PP. The situation of the elderly in Chile. Rev Med Chile 1998; 125: 1207-12.

62. Marín PP, Hoyl T, Gac H, Carrasco M, Duery P, Petersen $\mathrm{K}$, et al. Assessment of 1497 Chilean nursing home residents, using the Resource Utilization Group method, RUG T-18. Rev Med Chile 2004; 132: 701-6. 